\title{
Empowering and Integrating Senior Citizens with Virtual Coaching
}

\author{
Andreas Braun ${ }^{1}$, Peter H.M.P. Roelofsma ${ }^{2}$, \\ Dieter Ferring ${ }^{3}$, and Milla Immonen ${ }^{4}$ \\ 1 Fraunhofer Institute for Computer Graphics Research IGD, Darmstadt, Germany \\ andreas.braun@igd.fraunhofer.de \\ 2 VU University, CAMeRA, Amsterdam, The Netherlands \\ p.h.m.p.roelof sma@vu.nl \\ 3 Université de Luxembourg, Luxembourg \\ dieter.ferring@uni.lu \\ 4 VTT Technical Research Centre of Finland, Oulu, Finland \\ milla.immonen@vtt.fi
}

\section{Background of the Workshop}

With Europes aging population and an increasing number of older people living alone or geographically distant from kin, loneliness is turning into a prevalent issue. This might involve deleterious consequences for both the older person and society, such as depression and increased use of healthcare services. Virtual coaches that act as friend in a para-social relationship but also as mentor that helps the elderly end- user to create meaningful relationships in his actual social environment are a powerful method to overcome loneliness and increase the quality of life in the elderly population. The AAL Joint Programme projects A2E2 (AAL-2008-1-071) and V2me (AAL-2009-2-107) are exploring virtual coaches and their application in AAL scenarios, including the use of user avatars, virtual self-representations that allow the user to be represented in communication scenarios. Other European research projects that focus on social integration of the elderly are e.g. ALICE (AAL-2009-2-091) or WeCare (AAL-2009-2-026). Outside the European Union the negative implications of population aging can be observed in Japan, having an even larger proportion of senior citizens, using individual-centred devices, such as robot pets 1 to improve the quality of life of lonely elderly persons.

The user groups involved often are not acquainted with modern ICT systems and therefore it is a challenge to create intuitive, adaptive platforms that cater to the individual needs and allow the user to interact easily.

\section{Aim of the Workshop}

The workshop will discuss the effects of virtual coaches on elderly users and how they can be used to improve the quality of life by aiding in planning daily life

\footnotetext{
${ }^{1}$ Wada, K.; Shibata, T., Living With Seal Robots - Its Sociopsychological and Physiological Influences on the Elderly at a Care House, IEEE Transactions on Robotics, Oct. 2007.
} 
activities and mediating meaningful relationships to maintain and expand the social network of the elderly persons. Additional applications of virtual coaches and avatars in AAL specific context will be discussed. Furthermore it will explore intuitive interaction between the user and virtual entities, leading to the following collection of topics:

1. Realistic virtual characters in AAL applications

2. Adaptive virtual self-representation in AAL applications

3. Emotional expressiveness of virtual characters

4. Intuitive interaction devices for elderly end-users

5. User interface design for interaction with virtual entities

6. Virtual entities in smart, sensor-equipped environments

7. Mediating social contacts by means of virtual coaching

8. Technology use and aging: Inhibiting and facilitating factors

9. User experience and acceptance evaluation: Results of pilot studies and prototype testing from end-user perspective

\section{Organisation of the Workshop}

The full-day workshop consists of two parts. In the first part, the different approaches to above topics are collected to create a set of current solutions, dealing with the socio-psychological and technical aspects of virtual coaches in AAL environments. In the second part, a forum will provide workshop participants with the possibility to evaluate the solutions collected in the first part to find common aspects and determine further applications scenarios for virtual characters in AAL. The workshops aims at creating a shared set of knowledge gathering the current state of virtual coaching for elderly persons and collecting novel approaches for further applications in AAL context.

The workshop is aimed at experts in ambient assistant living services, sociopsychological methods, usability engineering and smart-environments. Psychologists, researchers and engineers working on the implementation of technical platforms, as well as usability and evaluation studies are called to join us in this workshop.

Each participant is expected to submit a position paper of 2-4 pages, describing experiences and results in research on the area of application scenarios of virtual coaches and virtual self-representations, intuitive interaction with virtual characters and integration of these into smart environments. 